\title{
Dosimetric Comparison of Stereotactic Body Radiotherapy and Intensity- Modulated Radiotherapy to Deliver Hypofractionated Radiotherapy in Organ- Confined Prostate Cancer
}

\author{
Mahadev Potharaju ${ }^{1}$, Murali Venkatraman ${ }^{1}$, Kurup Gopalakrishna ${ }^{1}$ \\ 1. Department of Radiation Oncology, Apollo Hospitals
}

$\square$ Corresponding author: Mahadev Potharaju, mahadev62@gmail.com

Disclosures can be found in Additional Information at the end of the article

\section{Abstract}

We compared the dose conformity, homogeneity, dose to organs at risk, and remaining volume at risk of two radiotherapy techniques --stereotactic body radiotherapy and intensitymodulated radiotherapy (IMRT) to deliver hypofractionated radiotherapy in organ-confined prostate cancer. Eleven successive patients treated on the CyberKnife stereotactic robotic radiosurgical system were taken up for this study. The CT images, including the structures contoured, were imported through DICOM RT from the Multiplan (Accuray Inc., Sunnyvale, CA, USA) treatment planning system to the Plato Sunrise treatment planning system (Nucletron, The Netherlands) for creating IMRT treatment plans on the Linac. Treatment planning was done for five fractions, each fraction prescribed to a dose of $7.25 \mathrm{~Gy}$ to the planning target volume. Dose volume histograms were generated for the CyberKnife and Linac treatments for comparison. As per our protocol, a plan was considered adequate if at least 95\% of planning target volume received the prescribed dose and satisfied the following constraints: a) volume of the rectum receiving 36Gy (V36) to < 1cc and b) volume of the bladder receiving 37Gy (V37) to < 10cc. Both the treatment techniques plans resulted in adequate planning target volume coverage ( $p$ value for D95, D90, D5, and V100\% was 0.402, 0.97, 0.013, and 0.012, respectively). CyberKnife plans were more conformal ( $p$ value 0.001). Remaining volume at risk dose was significantly less in all CyberKnife plans at [V100\% ( $\mathrm{p}=0.01), \mathrm{V} 95 \%(\mathrm{p}=.004), \mathrm{V} 90 \%(\mathrm{p}=.001)$, V80\% ( $\mathrm{p}=0.02)$, V70 ( $\mathrm{p}=0.03), \mathrm{V} 40 \%(\mathrm{p}=0.021)$, and V30\% ( $\mathrm{p}=0.001)]$ dose levels. The mean dose to rectum was lower in the CyberKnife plans (The p value for V100\%, V80\%, V50\% and V30\% dose levels was $0.02,0.0001,0.0002$, and 0.12 , respectively).

Received 12/29/2012 Review began 01/01/2013 Published 02/21/2013

\section{(c) Copyright 2013}

Potharaju et al. This is an open access article distributed under the terms of the Creative Commons Attribution License CC-BY 3.0., which permits unrestricted use, distribution, and reproduction in any medium, provided the original author and source are credited.
Categories: Radiation Oncology, Urology

Keywords: prostate, cancer, imrt, cyberknife, sbrt, hypofractionation

\section{Introduction}

The treatment of organ-confined prostate cancer by external radiotherapy has evolved over a period of time from the conventional two-dimensional to high precision techniques, like 3D conformal radiotherapy, intensity-modulated, and image-guided radiotherapy. The main advantage of these high precision radiation techniques has been the potential for dose escalation in the range of 76-80 Gy without experiencing any undue rectal or bladder morbidity. There are several randomized Phase 3 trials demonstrating significant improvement in five years outcomes in organ-confined prostate cancer at these escalated doses [1-2]. 
The conventional fractionation used in intensity-modulated radiotherapy (IMRT) is usually 180-200 cGy/fraction, five fractions a week delivered over a period of around eight weeks. This has logistic implications in terms of work productivity and the cost of the treatment. Hypofractionation is an attractive concept in prostate cancer. The treatment is over in a short time, resulting in significant socioeconomic benefits for the patient. The radiobiologic rationale for hypofractionation in prostate cancer is the hypothesis that prostate cancer has a low $\alpha / \beta$ ratio (1.5-3), as suggested by various authors [3-5]. Hypofractionation in prostate cancer has been practiced as early as in 1993 when the present sophisticated radiotherapy techniques were not available. Two hundred and thirty-two prostate cancer patients were treated at London with a fractionation schedule of 6x6Gy with impressive results and without any undue morbidity [6]. Manchester reported their experience with a hypofractionated schedule of 15x $3.1 \mathrm{~Gy}$ [7]. Hypofractionation in prostate cancer can be delivered either by external radiotherapy, i.e. 3-D conformal radiotherapy, IMRT, stereotactic body radiotherapy (SBRT), or high dose rate (HDR) brachytherapy [8].

There are reports of hypofractionation schedules with IMRT as well, showing the potential of delivering high doses per fraction without undue morbidity [9]. There has been also interest in SBRT in organ-confined prostate cancer, based mainly on the encouraging results obtained with high-dose-rate brachytherapy. Stereotactic body radiotherapy aims to deliver large doses per fraction to the target volume, while minimizing the dose to the surrounding critical normal structures. Improvements in image guidance tools, particularly frequent online tracking, have made delivery of stereotactic body radiotherapy feasible, reliable and safe.

The CyberKnife is one such machine among others available to deliver SBRT. Different institutions follow different image guidance protocols in the delivery of IMRT. At our institution, daily KV cone beam CT is done everyday before starting the radiotherapy. However, hypofractionation by no means can be considered the standard of care in the management of organ-confined prostate cancer. The ongoing PACE multi-institutional trial will probably be able to throw more light on this. This study is an international multicenter randomized study of organ-confined low and intermediate risk prostate cancer and is composed of two parallel randomization schemes based on applicability of surgery as a treatment for the patient. Patients for whom surgery is a consideration are randomized to either laparoscopic or da Vinci prostatectomy or CyberKnife prostate SBRT. Patients for whom surgery is not a consideration are randomized to either conventionally fractionated radiation therapy or CyberKnife prostate SBRT. Efficacy, toxicity and quality of life outcomes will be compared across the pairs in each randomization.

\section{Materials And Methods}

Traditionally, treatment planning systems for IMRT in prostate cancer use seven to nine isocentric non-opposed beams. The CyberKnife Multiplan treatment planning system uses non-isocentric beams that could be directed from innumerable different angles to deliver such treatments. Both types of planning system use inverse planning software. IMRT plans for the step-and-shoot technique were done using Plato Sunrise planning system, version $2.7 \mathrm{v}$ (Nucletron B.V, The Netherlands). The inverse planning optimization algorithm used is Konrad Optimization developed by MRC system, Heidelberg, Germany using gradient search method. The absolute dose calculation is based on the pencil beam dose calculation algorithm developed by Bortfield, et al. [10]. The algorithm calculates the dose using the percentage depth dose (PDD) for the field size at source to skin distance (SSD), the off-axis ratio (OAR) and the output factor. For in-homogeneity correction, equivalent path length method is employed. The CyberKnife plans done with Multiplan planning system version 3.5.4 (Ms. Accuray, Sunnyvale, USA) uses sequential optimisation programme. The sequential optimization programme differs from the conventional optimization, where multiple objectives are grouped in a single cost function and optimised simultaneously. The sequential optimization is executed sequentially 
as a series of individual optimization steps. The optimization steps are meant for target conformality, minimisation of maximum or mean dose to the OAR and minimising MU. The multiplan dose calculation is based on a ray-racing algorithm, which basically uses beam parameters namely, tissue phantom ratio (TPR), off-centre ratio (OCR) and the output factor, that are stored in the system [11]. For in-homogeneity correction effective path length along the central axis is used. The dose calculation algorithm approach is the same for both the planning systems. Hence, it can be mentioned that the difference in final dose distribution is solely due to the optimization approach and the resultant beamlets (which again depends on the mechanical constraints of the treatment machine), as same goals are set for the target coverage and OAR, in both the planning systems.

For patients undergoing CyberKnife planning, four gold fiducials were placed in the prostate transperineally or transrectally. A week later, planning CT and MRI were done without a bladder catheter. Real time tracking during treatment was done with the help of roof-mounted $\mathrm{x}$-ray imaging sources which take orthogonal images either for every beam or at some specified intervals set in the system for verifying the fiducials position. The position of these fiducials was automatically registered with the digitally reconstructed radiographs from the planning CT for online tracking of intrafractional translational and rotational movements of the prostate. A dose of $36.25 \mathrm{~Gy}$ in five fractions was prescribed to the PTV. The PTV was defined as the prostate with $5 \mathrm{~mm}$ margin all around, excluding the seminal vesicles. The dose-volume constraints used were to limit

a) Volume of the rectum receiving 36Gy (V36) to < 1cc

b) Volume of bladder receiving 37Gy (V37) to < 10cc.

The Multiplan treatment planning system uses iterative or sequential optimization using either a combination of fixed collimators or the Iris variable aperture collimator (Accuray Incorporated, Sunnyvale, CA, USA) for generating a suitable treatment plan. The advantage of Iris collimator is that the field size can be varied automatically during treatment, and hence saves considerable time in contrast to the use of fixed collimators where the robotic manipulator has to pick up the desired collimator from the stand when multiple size collimators are used for planning. The plan quality, however, does not depend upon the whether iris collimator or fixed collimators are used. IMRT plans were done on the same CT slices (with the same contoured structures) that were used for CyberKnife plans. The comparative IMRT treatment plans were done using the same dose volume constraints and using seven co-planar non-opposed beams. The maximum number of segments allowed were 70 for the IMRT plans as per our institutional protocol.

The dose to the target volume, organs at risk (rectum and bladder), remaining volume at risk (RVR), conformation number, and homogeneity indices were compared for both the treatment techniques. Statistical analysis was performed using a paired two-tailed student $t$ test to examine any statistically significant difference in the parameters examined. The $p$ value $<0.05$ was considered statistically significant.

\section{Comparison parameters}

For comparison purposes, all CyberKnife and IMRT plans were normalized, such that $95 \%$ of the planning target volume received at least $95 \%$ of the prescribed dose. Dose volume histograms were generated for each plan. The following dosimetric endpoints were evaluated for planning target volume coverage: V100\%, D5, D90, D95. Vx is defined as the percentage of the normalized volume of the region of interest irradiated by $\mathrm{x} \%$ of the prescribed dose. Dx is the dose coverage at $\mathrm{x} \%$ of the PTV respectively. 
Conformation number [12] and homogeneity index were used to evaluate the conformity and the homogeneity of target doses.

Conformation number $(\mathrm{CN})$ is defined as:

$C N=\frac{V T_{r e f}}{V T} \times \frac{V T_{r e f}}{V_{r e f}}$

Where VT ref = volume of target receiving a dose equal to or greater than the reference dose, VT $=$ volume of target, $\mathrm{V}$ ref $=$ volume receiving a dose equal to or greater than the reference dose (treated volume). The conformation number varies between 0 and 1 . A higher value means a better conformality. This is in contrast to the conformity index that is a ratio of the prescription isodose volume over the tumor volume. The conformation number considers, in addition, the volume of normal tissue getting a dose equal to greater than the reference dose.

Homogeneity index (HI) was determined from the following equation:

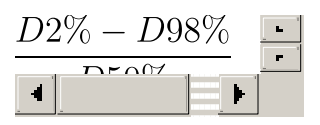

Ideal value is 0 for $\mathrm{HI}$.

The conformity difference ratio (CN\%) and homogeneity difference ratio (HI\%) was calculated by the following equations respectively:

$$
\begin{aligned}
& C N \%=\frac{C N C K-C N I M R T}{C N I M R T} \times 100 \\
& H I \%=\frac{H I C K-H I I M R T}{H I I M R T} \times 100
\end{aligned}
$$

These two parameters can be used to quantify the improvement or reduction in the CN \& HI, respectively.

For the organs at risk (OAR's), the bladder and rectal exposure (V30\%, V50\%, V80\%, V100\%) of each patient in each plan was evaluated. In addition, the dose to the RVR was also evaluated.

Remaining volume at risk: As per ICRU (International Commission on Radiation Units and Measurements) report 83, RVR is defined by the difference between the volume enclosed by the external contour of the patient and that of the CTV and OAR's on the slices that have been imaged. This is a measure of all the normal tissues that could potentially receive significant radiation.

Comparing the mean value differences of the CK and IMRT plans, calculations of statistical significance were performed using the paired $t$ test.

\section{Results}

\section{Planning target volume coverage}

Table 1 gives the dose volume parameters for clinical target volume taken from the DVH's (dose 


\section{Cureus}

volume histograms) of the CyberKnife and corresponding IMRT plans for all the patients. The CyberKnife plans, in general, produced a better planning target volume coverage than the IMRT plans. The p value was statistically significant, (0.012 \& 0.013), for the average V100\% and D5. However, the p value for D 90 and D95 was 0.97 and 0.42 , showing no statistically significant difference in coverage at these levels.

\begin{tabular}{|c|c|c|c|}
\hline Parameter & CK SBRI Plan(\%) & IMRI Plan(\%) & p value \\
\hline D95 & $98.8+-2.74$ & $97.7+-2.99$ & 0.42 \\
\hline D90 & $101.6+-2.26$ & $101.7+-2.45$ & 0.97 \\
\hline D5 & 113.3+-3.96 & $110.1+-1.90$ & 0.013 \\
\hline V100\% & $58.6+-18.73$ & $56.69+-17.73$ & 0.012 \\
\hline
\end{tabular}

\section{TABLE 1: Dose volume parameters for CK SBRT and IMRT for planning target volume}

Statistically significant difference is displayed in bold (paired two-tailed t test) Abbreviations: CK=CyberKnife, IMRT=Intensitymodulated radiotherapy, SBRT = Stereotactic body radiotherapy. Dx is the dose coverage at $\mathrm{x} \%$ of the PTV, Vx = percentage of the normalized volume of the region of interest irradiated by $\mathrm{x} \%$ of the prescribed dose

\section{Dose to organs at risk}

Table 2 elucidates the dose volume parameters for rectum and bladder for the two treatment modalities. There is more efficient rectal sparing in the CyberKnife plans, which is statistically significant at V100\%, V80\%, and V50\% dose levels. The p value was 0.02, 0.0001, and 0.0002, respectively. 


\section{Cureus}

\begin{tabular}{|c|c|c|c|}
\hline & CK SBRI & IMRT & p value \\
\hline \multicolumn{4}{|l|}{ Rectum } \\
\hline V100\% & $0.07 \pm 0.07$ & $1.2 \pm 1.34$ & 0.02 \\
\hline V80\% & $3.4 \pm 2.75$ & $9.2 \pm 4.79$ & 0.0001 \\
\hline V50\% & $15.5 \pm 7.24$ & $30.1 \pm 11.94$ & 0.0002 \\
\hline V30\% & $32.7 \pm 12.34$ & $35.7 \pm 12.02$ & 0.12 \\
\hline \multicolumn{4}{|l|}{ Bladder } \\
\hline V100\% & $1.3 \pm 2.28$ & $2.6 \pm 2.3$ & 0.08 \\
\hline V80\% & $10.4 \pm 6.84$ & $10.3 \pm 6.10$ & 0.92 \\
\hline V50\% & $38.6 \pm 16.54$ & $33.8 \pm 15.02$ & 0.23 \\
\hline V30\% & $85 \pm 30.55$ & $53.7 \pm 25.04$ & 0.006 \\
\hline
\end{tabular}

\section{TABLE 2: Dose volume parameters for OAR (organs at risk)}

Statistically significant difference is displayed in bold (paired two-tailed t test). Abbreviations as in Table 1

In contrast, there is no appreciable difference in bladder sparing between the CyberKnife and the IMRT plans at these dose levels (V100\%, V80\%, and V50\%) for which the corresponding $\mathrm{p}$ values were $0.08,0.92$, and 0.23 , respectively. However, in the low dose regions of V30\%, volume of bladder receiving this dose is higher in the CyberKnife plans as compared to the simulated IMRT plans ( $\mathrm{p}=0.006$ ). The lower dose to the rectum in the CyberKnife plans reflects a much sharper fall-off of dose posteriorly when compared to the IMRT plans. This could be because most of the beams in the CyberKnife plans are from anterior and lateral direction and the use of a large number of non-coplanar beams. This is a distinct advantage as rectal morbidity is one of the limiting factors in delivering hypofractionated radiotherapy. Our results are in accordance with a similar study done by Sabbir Hosaain, et al. and Ceylan, et al. [12-13] comparing these two modalities (CyberKnife and IMRT). However, in a study done by Shiu, et al. comparing CyberKnife and IMRT dose distributions, they found the dose to critical organs were less in the IMRT plans [14]. 


\section{Cureus}

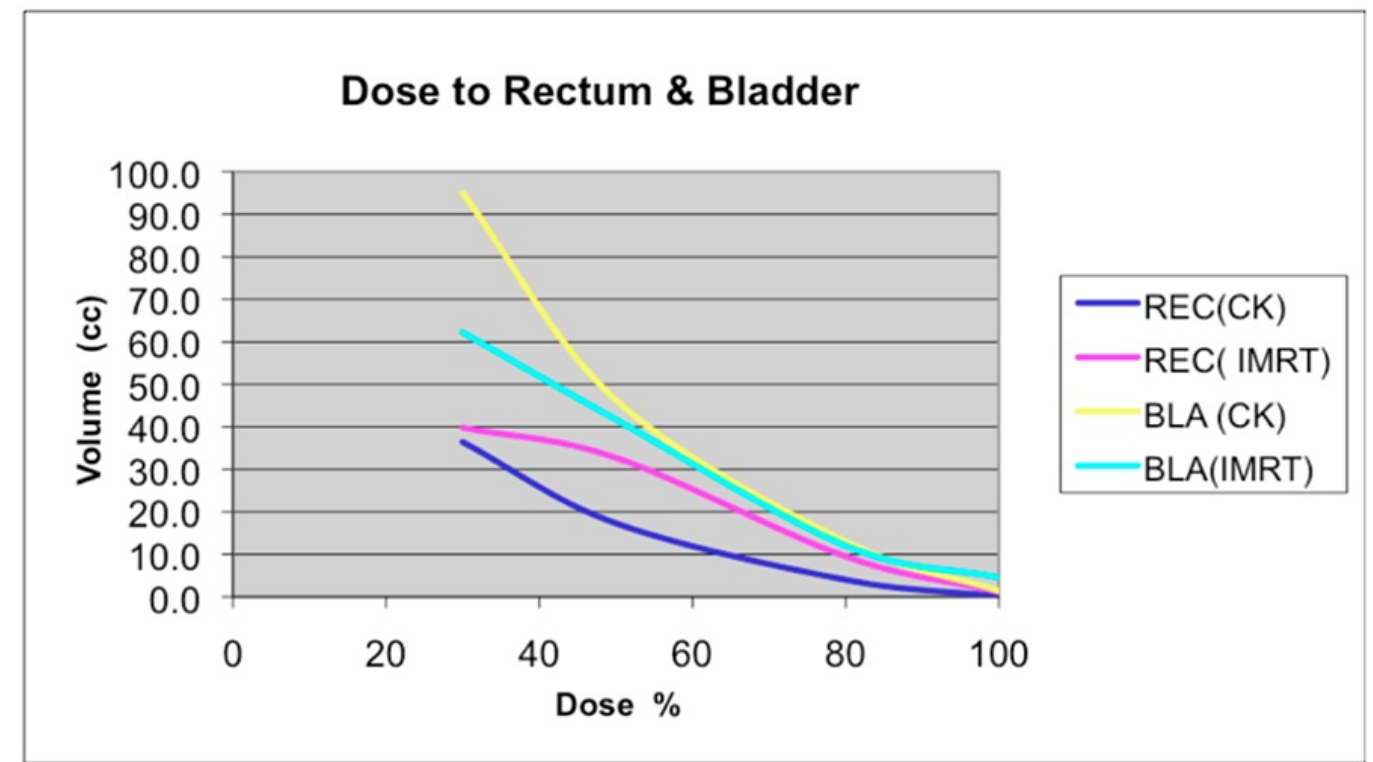

FIGURE 1: Dose to Rectum \& Bladder

Remaining volume at risk dose

Table 3 gives the dose volume histogram values for remaining volume at risk. 


\section{Cureus}

\begin{tabular}{|llll|}
\hline RVR & CK (cc) & IMRT (cc) & p value \\
\hline V100\% & $11.5 \pm 6.6$ & $17.2 \pm 6.4$ & 0.011 \\
V95\% & $24.4 \pm 11.01$ & $33.4 \pm 11.03$ & 0.004 \\
V90\% & $39.2 \pm 14.32$ & $50.6 \pm 15.42$ & 0.001 \\
V80\% & $67.4 \pm 22.26$ & $79.7 \pm 22.42$ & 0.02 \\
V70\% & $99.7 \pm 30.04$ & $118.2 \pm 35.76$ & 0.03 \\
V60\% & $146.8 \pm 45.81$ & $178 \pm 54.42$ & 0.012 \\
V50\% & $230 \pm 79.27$ & $289.9 \pm 102.41$ & 0.008 \\
V40\% & $431.3 \pm 163.5$ & $596.7 \pm 231.6$ & 0.01 \\
V30\% & $877.5 \pm 316.4$ & $1514.8 \pm 576.46$ & 0.0005 \\
V20\% & $1793 \pm 630.25$ & $2075.9 \pm 683.6$ & 0.1 \\
\hline
\end{tabular}

TABLE 3: Comparison of dose volume statistics of remaining volume at risk in CK SBRT and IMRT plans

Statistically significant difference is displayed in bold (paired two-tailed t test) Abbreviations: RVR= Remaining volume at risk, other abbreviations as in Table1

RVR is less in all CyberKnife plans compared to the simulated IMRT plans reaching statistical significance at $\mathrm{V} 100 \%(\mathrm{p}=0.01), \mathrm{V} 95 \%(\mathrm{p}=0.004), \mathrm{V} 90 \%(\mathrm{p}=0.001), \mathrm{V} 80 \%(\mathrm{p}=0.02), \mathrm{V} 70 \%(\mathrm{p}=0.03)$, $\mathrm{V} 60 \%(\mathrm{p}=0.01), \mathrm{V} 50 \%(\mathrm{p}=0.01), \mathrm{V} 40 \%(\mathrm{p}=0.02)$, and $\mathrm{V} 30 \%(\mathrm{p}=0.001)$ levels indicating lower nonclinical target volume, non-organ at risk tissue exposure at these dose levels. This has important clinical implications. When IMRT started gaining popularity in clinical use, one of the major concerns was that, even though there was significant dose reduction to the critical structures, larger volumes of normal tissue were exposed to the low-dose regions which could have the potential of inducing second malignancies [15-16]. This is thought to be mainly due to the increased monitor units and more number of fields used in IMRT. The second malignancies are a concern in long-term survivors. One would expect a majority of the organ-confined prostate cancer patients to be cured completely, and hence would probably live for a reasonably long time. Therefore, it is desirable to keep the normal volume low-dose radiation regions as less as possible. In this respect, the CyberKnife plans with lower remaining volume at risk doses have a slight edge over the IMRT plans.

\section{Dose to testes}

The mean dose to the testes was evaluated retrospectively. The testes were not contoured at the time of planning, and hence no attempt was made to limit the dose to testes. The mean dose was $142.5 \mathrm{cGy}$ and $45 \mathrm{cGy}$ in the CyberKnife and IMRT plans respectively, which was statistically significant $(\mathrm{p}=0.001)$

\section{Conformation number, homogeneity index, conformity}




\section{Cureus}

\section{difference ratio, and homogeneity difference ratio}

Table 4 lists the mean and standard deviation of the conformation number, homogeneity index, conformity difference ratio, and homogeneity difference ratio values for each patient in the CyberKnife and IMRT plans.

\begin{tabular}{|c|c|c|c|c|c|c|c|}
\hline Patient no. & PTV in cc & CK & CN IMRT & $! C N \%$ & CK & HI IMRT & !HI\% \\
\hline 1 & 37.86 & 0.6729 & 0.5479 & 22.81 & 0.2309 & 0.2272 & 1.6 \\
\hline 2 & 44.15 & 0.8242 & 0.7878 & 4.62 & 0.1516 & 0.2148 & -29.4 \\
\hline 3 & 45.37 & 0.7597 & 0.6876 & 10.48 & 0.1976 & 0.1374 & 43.81 \\
\hline 4 & 56.87 & 0.7866 & 0.6485 & 21.29 & 0.1789 & 0.1194 & 49.83 \\
\hline 5 & 70.32 & 0.7866 & 0.6951 & 13.23 & 0.2061 & 0.1436 & 43.52 \\
\hline 6 & 75.2 & 0.8726 & 0.7481 & 16.64 & 0.0933 & 0.1192 & -21.7 \\
\hline 7 & 75.9 & 0.7239 & 0.676 & 7.08 & 0.1691 & 0.1923 & -12.1 \\
\hline 8 & 77 & 0.7355 & 0.681 & 8 & 0.1433 & 0.1414 & 1.34 \\
\hline 9 & 90.08 & 0.9365 & 0.762 & 22.9 & 0.1269 & 0.174 & -27.1 \\
\hline 10 & 91.2 & 0.8289 & 0.7977 & 3.91 & 0.198 & 0.1203 & 64.58 \\
\hline 11 & 167 & 0.9144 & 0.8222 & 11.21 & 0.2416 & 0.1554 & 55.47 \\
\hline Mean & 75.54 & 0.804 & 0.714 & 12.6 & 0.1761 & 0.1586 & 11.03 \\
\hline SD & 35.37 & 0.081 & 0.079 & 2.53 & 0.045 & 0.0384 & 17.2 \\
\hline p value & & & 0.0001 & & & 0.305 & \\
\hline
\end{tabular}

\section{TABLE 4: Conformation number and homogeneity index values for each patient}

Statistically significant difference is displayed in bold (paired two-tailed $t$ test) Abbreviations: $C N=$ conformation number, !CN\%= Conformity difference ratio, $\mathrm{HI}=$ Homogeneity index, $! \mathrm{HI} \%=$ Homogeneity difference ratio, $\mathrm{SD}=\mathrm{Standard}$ deviation, no.=number 
The average homogeneity index value and standard deviation for the CyberKnife plans was $0.1768 \pm 0.045$ and $0.159 \pm 0.039$ for the IMRT plans, suggesting that CyberKnife plans are slightly more inhomogenous than the IMRT plans but statistically not significant $(\mathrm{p}=.305)$. The average homogeneity difference ratio value was $11.03 \pm 17.2$. In five patients, the IMRT plans were more inhomogenous than the CyberKnife plans, and in the remaining seven patients, the CyberKnife plans were more inhomogenous when compared with the corresponding IMRT plans [14].

\section{Discussion}

$\mathrm{N} / \mathrm{A}$

\section{Conclusions}

IMRT and CyberKnife SBRT can be used to deliver hypofractionated schedules in organconfined prostate cancer satisfying the specified constraints for organs at risk. Both treatment techniques provide adequate PTV coverage. In our study, CyberKnife plans delivered reduced doses to rectum and remaining volume at risk, which was statistically highly significant. CyberKnife plans are more conformal than IMRT plans, which was statistically significant. Though the CyberKnife plans are more inhomogenous than the IMRT plans, it does not reach statistical significance. Both these techniques are viable alternatives to HDR brachytherapy to deliver a hypofractionated schedule in a non-invasive way. However, precise image guidance tools are required to deliver these hypofractionated regimens. Randomized trials comparing conventional fractionation and hypofractionation are necessary to determine the ideal fractionation schedule. The results of the ongoing PACE study may provide more insight into this issue. Hence, long-term data is required before stereotactic body radiotherapy can be considered as an alternative to conventional fractionation in organ-confined prostate cancer.

\section{Additional Information}

\section{Disclosures}

Human subjects: All authors have confirmed that this study did not involve human participants or tissue. Animal subjects: All authors have confirmed that this study did not involve animal subjects or tissue. Conflicts of interest: In compliance with the ICMJE uniform disclosure form, all authors declare the following: Payment/services info: All authors have declared that no financial support was received from any organization for the submitted work. Financial relationships: All authors have declared that they have no financial relationships at present or within the previous three years with any organizations that might have an interest in the submitted work. Other relationships: All authors have declared that there are no other relationships or activities that could appear to have influenced the submitted work.

\section{References}

1. Pollack A, Zagars GK, Starkschall G et al: Prostate cancer radiation dose response: results of the M.D.Anderson phase 3 randomised trial. Int J Radiat Oncol Biol Phys. 2002, 53:1097. 10.1016/S0360-3016(02)02829-8

2. Zietman AL, De Silvio ML, Slater JD et al: Comparison of conventional dose vs high-dose conformal radiotherapy in clinically localized adenocarcinoma of the prostate: a randomized controlled trial. JAMA. 2005, 294:1233. 10.1001/jama.294.10.1233

3. Fowler JF, Chapell R, Ritter M: Is $\alpha / \beta$ for prostate tumors really low? . Int J Radiat Oncol Biol Phys. 2001, 50:1021-1031. 10.1016/S0360-3016(01)01607-8

4. Brenner DJ, Hall EJ: Fractionation and protraction for radiotherapy of prostate carcinoma . Int J Radiat Oncol Biol Phys. 1999, 43:1095-1101.

5. GM, Peters LJ: What is the $\alpha / \beta$ ratio for prostate cancer? Rationale for hypofractionated highdose rate brachytherapy. Int J Radiat Oncol Biol Phys. 1999, 44:747-748. 
6. Collins CD, Lloyd-Davies RW, Swan AV: Radical external beam radiotherapy for localised carcinoma of the prostate using a hypofractionation technique. Clin Oncol (R Coll Radiol). 1991, 3:127-13. 10.1016/S0936-6555(05)80831-3

7. Logue JP, Cowan RA, Hendry JH: Hypofractionation for prostate cancer. Int J Radiat Oncol Biol Phys. 2001, 49:1522-1523.

8. Yoshioka Y, Konishi K, Sumida I, Takahashi Y et al: Monotherapeutic High-Dose-Rate Brachytherapy for Prostate Cancer: Five-Year Results of an Extreme Hypofractionation Regimen With 54 Gy in Nine Fractions. Int J Radiat Oncol Biol Phys. 2011, 80:469-475. 10.1016/j.ijrobp.2010.02.013

9. Amer AM, Mott J, Mackay RI et al: Prediction of the benefits from dose-escalated hypofractionated intensity-modulated radiotherapy for prostate cancer. Int J Radiat Oncol Biol Phys. 2003, 56:199-207. 10.1016/S0360-3016(03)00086-5

10. Bortfield T, Schlegel W and Rhein B: Decomposition of pencil beam kernels for fast dose calculations in three-dimensional treatment planning. Med. Phys. 1993, 20:311-318.

10.1118/1.597070

11. Schlaefer A, Schweikard A: Stepwise multi-criteria optimization for robotic radiosurgery. Med Phys. 2008, 35:2094-2103. 10.1118/1.2900716

12. Van’t Riet A, Mak AC, Moerland MA, Elders LH, van der Zee W: A conformation number to quantify the degree of conformality in brachytherapy and external beam irradiation: application to the prostate. Int J Radiat Oncol Biol Phys. 1997, 37:731-6. 10.1016/S03603016(96)00601-3

13. Hossain S, Xia P, Huang K, Descovich M, Chuang C, Gottschalk AR, Roach M 3rd, Ma L: Dose Gradient Near Target-Normal Structure Interface for Nonisocentric CyberKnife and Isocentric Intensity-Modulated Body Radiotherapy for Prostate Cancer. Int J Radiat Oncol Biol Phys. 2010, 78:58-63. 10.1016/j.ijrobp.2009.07.1752

14. Ceylan C, Kucuk N, Avata HB, Guden M, Engin K: Dosimetric and physical comparison of IMRT and cyberknife plans in the treatment of localized prostate cancer. Reports of Practical Oncology \& Radiotherapy. 2010, 15:181-189.

15. Shiu A, Wang CH, Ye J, Cotrutz C, Meier R, Mehta V, Vermeulen S, Chang EL: Dose Distribution Comparison for the IGRT Treatment Using CyberKnife Versus LINAC IMRT Stereotactic Body Radiotherapy. Int J Radiat Oncol Biol Phys. 2007, 69(3) Supplement:S689.

16. Hall EJ, Wuu CS: Radiation-induced second cancers: the impact of 3D-CRT and IMRT . Int J Radiat Oncol Biol Phys. 2003, 56:83-8. 10.1016/S0360-3016(03)00073-7 